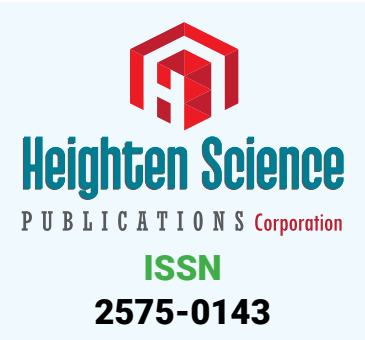

*Address for Correspondence: Ahmed Zaky, $\mathrm{MD}, \mathrm{MPH}, \mathrm{FCCM}$, University of Alabama at Birmingham, Birmingham, USA, Tel: 3522176319 Email: azaky@uabmc.edu; drazaky@yahoo.com

Submitted: 31 October 2018

Approved: 20 November 2018

Published: 21 November 2018

Copyright: (c) 2018 Zaky A. This is an open access article distributed under the Creative Commons Attribution License, which permits unrestricted use, distribution, and reproduction in any medium, provided the original work is properly cited

D) Check for updates
Commentary

\section{Is there an ideal blood pressure during cardiopulmonary bypass to prevent postoperative cerebral injury? - What does the recent evidence say?}

\author{
Ahmed Zaky* \\ University of Alabama at Birmingham, Birmingham, USA
}

\section{Background}

Post cardiac surgery stroke is a devastating complication with an incidence as high as $50 \%{ }^{1}$. The association between intra-operative mean arterial blood pressure (MAP- better called linear blood pressure) during cardiopulmonary bypass (CPB) and the development of postoperative stroke-as diagnosed by neuroimaging- and of cognitive dysfunction (POCD) is controversial. This is due to differences in the study populations, stroke assessment tools, operation and conduction of MAPs, variations in neurocognitive testing and duration of follow up. As a result there is a gap in the knowledge on an ideal MAP as a preventive measure of post CPB stroke and POCD.

\section{Study Summary}

Study objective and hypothesis

To compare between a low MAP (40-50 mmHg) and high MAP (70-80 mmHg) strategies during CPB and the development of diffuse-weighted MRI (DWI)-diagnosed silent postoperative stroke. The authors hypothesized that a high MAP strategy will be associated with significantly less incidence of silent strokes compared to a low MAP strategy.

Design

Single-center, assessor/patient-blinded parallel, stratified randomized controlled study.

\section{Outcomes}

Primary: Volume of ischemic strokes as diagnosed by DWI-MRI on days 3 and 6 postoperatively compared to baseline.

\section{Secondary}

a. Total number of new strokes as diagnosed by DWI-MRI and

b. POCD was diagnosed by international Study postoperative cognitive dysfunction (IS-POCD) testing at 1 week, at discharge, and at 2 and 4 months postoperatively, as a change from baseline.

c. Occurrence of new postoperative focal neurological deficit at same time intervals as (b).

Study procedures

In the high MAP strategy, phenylephrine boluses were used up to maximum dose of 
$2 \mathrm{mg}$ followed by norepinephrine at a maximal dose of $0.4 \mathrm{mcg} / \mathrm{kg} / \mathrm{min}$. Pump flow was constant in both groups. CPB management guidelines were followed in both groups.

\section{Results}

A total of 197 patients were enrolled; $\mathrm{N}=99$ in the low- and N=98 in the high-MAP groups. The high MAP group was significantly older in age, yet no other baseline characteristics were different between both groups.

There were no differences in primary or secondary outcomes between low- and high MAP-strategy groups, with a statistically non-significant trend towards higher volume of ischemic infarcts and worse POCD at 30 days in the high MAP strategy group.

\section{Discussion}

The main findings of this study is a lack of a statistically significant differences in overt and covert strokes as well as POCD between a high and a low-MAP strategy in patients undergoing valve surgery under $\mathrm{CPB}$.

The major strengths of this study compared to others ${ }^{23}$ is that it achieved a baseline testing on all patients, so that changes in volume of covert stroke is confidently diagnosed as a 'new' onset. This study was also statistically powered for its primary and secondary outcomes based on a pilot study that preceded the conduction of this study. Besides, the study also used a sensitive imaging tool to diagnose covert strokes and, as well, assessed both covert and overt strokes and POCD.

The major limitations of this study is being a single study, lacking external validation, with a relatively heterogeneous study population and with a statistically significant baseline between-group differences in age, which may explain the trend towards higher volumes of ischemic strokes and worse POCD in patients with high MAP strategy. Another limitation is the relatively high doses of norepinephrine used in this study and the use of a different neurocognitive testing compared with previous studies. Additionally, the authors managed MAPs only during the CPB period with the possibility of blood pressure perturbations after CPB wean [1-3].

\section{Conclusion}

The debate continues on an ideal hemodynamic strategy to prevent post CPB stroke. At present, a high MAP is neither preventive nor protective. More studies are needed to investigate the influence of hemodynamics among other resuscitation modalities on the development of post CPB stroke.

\section{References}

1. Tarakji KG, Sabik JF, 3rd, Bhudia SK, Batizy LH, Blackstone EH. Temporal onset, risk factors, and outcomes associated with stroke after coronary artery bypass grafting. JAMA. 2011; 305: 381-390. Ref.: https://goo.gl/CVG9er

2. Gottesman RF, Sherman PM, Grega MA, Yousem DM, Borowicz LM, Jr., et al. Watershed strokes after cardiac surgery: diagnosis, etiology, and outcome. Stroke. 2006; 37: 2306-2311. Ref.: https://goo.gl/v3LZ88

3. Newman MF, Kirchner JL, Phillips-Bute B, Gaver V, Grocott H, et al. Longitudinal assessment of neurocognitive function after coronary-artery bypass surgery. $\mathrm{N}$ Engl $\mathrm{J}$ Med. 2001; 344: 395-402. Ref.: https://goo.gl/9ENmb1 